\title{
Dialogando sobre cultura de paz e bullying por meio de uma web rádio com alunos de escolas públicas de Picos, Piauí
}

\author{
Dialoguing about culture of peace and bullying through a web radio with \\ students from public schools of Picos, State of Piaui, Brazil
}

\section{RESUMO}

Objetivou-se descrever as experiências com a mobilização de estudantes de escolas públicas do município de Picos, Piauí, utilizando a tecnologia da Web Rádio AJIR (Associação dos Jovens do Irajá), como meio de transmissão do programa "Em Sintonia com a Saúde" sobre cultura de paz e bullying. Trata- se de um relato de experiência do projeto de extensão Web Cuidado em Infância e Juventude nas Escolas, com a transmissão da Web Rádio AJIR no mês de agosto de 2016, em uma escola de Picos com 20 alunos do oitavo ano do ensino fundamental. Sobre cultura e paz, verificou-se que as principais dúvidas estavam relacionadas em como construir a paz $(38,9 \%)$. Quanto ao bullying, o principal questionamento foi sobre o que fazer para que ele não aconteça (33,3\%). Concluímos que por meio de atividades como essa é possível despertar um contato direto com a juventude e observar a enorme ânsia no aprendizado quando se tem a valiosa associação entre tecnologias e educação.

Palavras-chave: Tecnologia. Educação em saúde. Adolescentes.

\section{ABSTRACT}

The aim of this study was to describe the experiences with the mobilization of students from public schools in the city of Picos, State of Piauí, Brazil, using the Web Radio AJIR technology, as a means of transmitting the program "In Tune with Health" about culture of peace and bullying. This is an experience report of the web extension project Children and Youth Care in Schools, with the transmission of the Web Radio AJIR in the month of August 2016 in a school of Picos with 20 students from the last year of elementary school. On culture and peace, it was verified that the main doubts regarded how to construct the peace $(38,9 \%)$. Concerning the bullying the main questioning was what to do to prevent it from happening $(33,3 \%)$. We conclude that through activities such as this one, it is possible to awaken a direct contact with the youth and observe the enormous eagerness in learning when one has this valuable association between
Eduardo de Oliveira Martins Dantas

Pós-graduando em Saúde Pública na Faculdade Entre Rios, Piauí, Brasil; integrante do projeto de extensão "Web cuidado em infância e juventude nas escolas”(eduardo8_oliveira@hotmail. com).

Victorugo Guedes Alencar Correia

Pós-graduando em Gestão em Saúde na Universidade Federal do Piauí, Brasil; integrante do projeto de extensáo "Web cuidado em infância e juventude nas escolas”(victorugoguedes@hotmail. com).

Marcos Renato de Oliveira

Doutor em Cuidados Clínicos pela Universidade Estadual do Ceará, Brasil; professor da Universidade Federal do Piauí, Brasil; coordenador do projeto de extensão "Web Cuidado em Infância e Juventude nas escolas". (enfmarcosrenato@hotmail.com).

Raimundo Augusto Martins Torres

Doutor em Educação pela Universidade Federal do Ceará, Brasil; professor da Universidade Estadual do Ceará, Brasil; responsável pela Web rádio AJIR (augusto.torres@uece.br). 
technologies and education.

Keywords: Technology. Health education. Adolescents.

\section{INTRODUÇÃO}

No decorrer do desenvolvimento tecnológico é observado um crescente aparecimento de recursos modernos que envolvem Tecnologias de Informação e Comunicação (TIC), e, portanto, está cada vez mais comum a sua utilização como meio de transmissão para a construção de conhecimentos e obtenção de saberes.

Dourado et al. (2014) afirmam que, no mundo atual, é impossível para a maioria da população viver sem a utilização de TIC, pois essas ferramentas, tais como televisão, jornal, web sites, internet, rádio, revistas e livros, difundem informaçóes que distribuem com ligaçóes, aproximando pessoas entre si.

Sendo assim, essa nova geração ainda demanda que haja modificaçóes na forma de ensinar e que mestres educadores aprimorem habilidades no manuseio de TIC, e também que a maioria dos países latinoamericanos as perceba como políticas públicas (HERRERA; FERNÁNDEZ; SEGUEL, 2018).

Correia e Santos (2013) relatam que o processo educacional vem passando por uma transformação no ensino, a qual influencia profundamente o desenvolvimento da educaçáo. Assim, o que antes era complemento, hoje é parte essencial, pois afeta de forma positiva não só o raciocínio de alunos e professores, como também o embasamento de sua tomada de decisóes.

As TIC também podem ser utilizadas em projetos de extensão universitária, em conjunto entre a universidade e a população, permitindo que haja troca de saberes entre ambas. Por meio dessa prática de troca, os acadêmicos recebem informaçóes sobre quais são os anseios de determinada comunidade e, a partir disso, podem realizar a implementação de atividades que visem à abordagem sobre as temáticas almejadas.

Naves (2015) menciona a extensão universitária como forma de aproximar a comunidade da Universidade, contribuindo, assim, 
para o progresso da produção e socialização do conhecimento. E, por outro lado, a comunidade pode ser beneficiada, a partir da absorçáo de saberes antes restritos apenas ao meio acadêmico.

A inserção dessas tecnologias no ambiente de aprendizado proporciona com mais facilidade as trocas de informaçóes entre as pessoas, pelo fato de ser uma forma que desperta o interesse dos adolescentes em aprender e também por ser um meio em que eles estão constantemente inseridos e capazes de dominar integralmente todas as atividades evolvendo tecnologias. Santos et al. (2017) dizem que o uso dos recursos tecnológicos de forma adequada é produtivo para o desenvolvimento intelectual, social e psicológico dos jovens e, portanto, é um fator progressivo e importante para a saúde.

Entretanto, na sociedade, sempre houve conflitos envolvendo a falta de tranquilidade e paz. Hodiernamente, nos deparamos com o aumento de casos de violência e preconceito, envolvendo o público jovem nas instituiçóes de ensino e aprendizagem. Araújo (2017) menciona que são diversos os tipos de violência e seus motivos. Sua prática, que está cada vez mais comum, geralmente ocorre em lugares com grande número de pessoas, como no caso dos ambientes escolares, em que vários educandos com comportamentos diferentes acabam por ocasionar desentendimentos. Entre esses problemas há o bullying, que tem como características o desequilíbrio de poder entre as pessoas com atos ofensivos intencionais repetitivos (OLWEUS, 2013), podendo ser ele físico ou virtual. E, para medidas preventivas e de resolução da violência, se tem a cultura de paz que estimula harmonia por meio da educação, comunicação e respeito mútuo (RUíZ, 2014).

Debater esses assuntos com mediaçóes tecnológicas no público adolescente é de alta relevância, pois Rocha et al. (2018) mencionam que intervençóes por meio de web rádio, tornam as atividades educativas mais atrativas, envolvendo o público e fazendo com que eles participem das atividades de forma interativa.

Assim, esta produção tem como objetivo descrever as experiências com a mobilização de estudantes de escolas públicas do município de Picos, Piauí, utilizando a tecnologia da Web Rádio AJIR (Associação dos Jovens do Irajá), como instrumento de transmissão do programa "Em Sintonia com a Saúde" sobre as temáticas: cultura de paz e bullying. 


\section{METODOLOGIA}

Trata-se de um estudo descritivo, do tipo relato de experiência do projeto de extensão Web Cuidado em Infância e Juventude nas Escolas (WEBCIJE) da Universidade Federal do Piauí (UFPI), realizado no mês de agosto de 2016, com abordagem quanti-qualitativa. Gil (2010) descreve os estudos descritivos como os que explanam características de determinada populaçáo e os de relato de experiência como a partilha de práticas de pesquisa e observação.

As atividades do projeto WEBCIJE aconteceram com encontros no ambiente escolar, da rede pública localizada em zona urbana na cidade de Picos, Piauí, no horário de transmissáo do programa "Em Sintonia com a Saúde" online por meio da Web Radio AJIR, vinculada ao Laboratório de Práticas Coletivas em Saúde (LAPRACS) da Universidade Estadual do Ceará (UECE).

Picos é um dos 224 municípios do estado do Piauí e está localizado a $320 \mathrm{~km}$ de distância da capital Teresina. Localiza-se na regiấo centrosul, fazendo parte da macrorregiáo 3: Semiárido, território do Vale do Guaribas. Tornou-se cidade no dia 12 de dezembro de 1890, estando atualmente com 127 anos de emancipação política. É atravessada pela BR-316 ou Rodovia Transamazônica, BR 407, e fica muito próxima a BR-020. Possui uma população estimada, em 2018, pelo Instituto Brasileiro de Geografia e Estatística (IBGE) de 78.002 habitantes (BRASIL, 2018).

A populaçáo desse estudo foi composta de alunos do oitavo ano do ensino fundamental de uma instituição estadual de ensino e a transmissão da rádio aconteceu às quartas-feiras, no período vespertino, no horário das $16 \mathrm{~h}$ às $17 \mathrm{~h}$, horário oficial da capital federal do Brasil ao vivo e direto de Fortaleza, capital do Ceará, a 531 km de distância de Picos, Piauí.

Primeiramente, antes dos encontros com os alunos, os integrantes do projeto realizaram leitura de artigos científicos como forma de obtenção de contato com conteúdos atualizados, que também eram debatidos em sala de aula. Entre os conteúdos estavam o estudo de Rocha, Costa e Passos Neto (2013) que explana o papel e a importância da sociedade junto com órgáos públicos no combate 
e prevenção do bullying, ressaltando que para ser considerada tal prática deve-se acontecer agressão intencional em ferir o alvo. E o de Cerdas-Agüero (2015) que menciona uma reflexão sobre os desafios que a educação enfrenta na construção de uma cultura de paz na América Latina.

Posteriormente, no ambiente escolar, as atividades procediam com a condução de um pequeno debate de ideias sobre as temáticas que seriam abordadas por meio da web rádio AJIR. Para um entretenimento mais dinâmico e motivador, as atividades iniciavam com uma pergunta sobre o tema, denominada de pergunta-âncora, e premiava-se quem a respondesse primeiro. Os/as jovens eram estimulados/as a fazerem suas perguntas/comentários para que fossem respondidas/comentados de maneira imediata por locutores e profissionais da área pela rádio.

O presente trabalho obteve aprovação do Comitê de Ética em Pesquisa da Universidade Estadual do Ceará (UECE) com o parecer de número: $11043817-5$ e seguiu os parâmetros preconizados pela Resoluçáo no 466/12 da Comissão Nacional de Ética em Pesquisa (CONEP), que dispóe sobre a pesquisa envolvendo seres humanos (BRASIL, 2012).

\section{RESULTADOS E DISCUSSÃO}

Os integrantes do projeto de extensão "Web Cuidado em Infância e Juventude nas Escolas" observaram que, por ser uma metodologia que foge das mediaçóes pedagógicas tradicionais de ensinar, a Web Rádio AJIR mostrou-se eficiente e satisfatória no modo de transmitir e motivar os jovens adolescentes, pois foi visto que eles estavam movimentados e participativos com a execução do projeto em sua escola.

Torres et al. (2015) mencionam que com a incorporação da potencialidade virtual visam-se técnicas inovadoras de transmitir saberes, ampliando a discussão sobre o uso de TIC, não só na educação, como também na promoção e prevenção da saúde, por meio da internet, pois o uso dessas práticas renovam o modo de se pensar e de fazer saúde. Para Mota et al. (2018), as TIC estáo presentes na vida cotidiana da população e facilitam a forma de transmissão de conteúdos de forma acelerada, requerendo que 
profissionais se apropriem desses recursos como forma facilitadora no desenvolvimento de suas competências.

Ainda, as atividades tiveram início com a leitura dos materiais, já mencionados na metodologia, como forma de introdução dos conteúdos a serem transmitidos, no qual prosseguiu a comunicação com a pergunta âncora que premiou o participante que a respondeu primeiro e de forma assertiva.

Com a explanação dos temas a serem abordados, verificou-se que os alunos levantaram questionamentos que foram enviados por meio de um aparelho celular e respondidos pelos apresentadores da web rádio AJIR, ao vivo, que se encontrava em Fortaleza. Durante o programa sobre cultura e paz, foi percebido, por meio da participação dos alunos com suas perguntas, que as principais dúvidas estavam relacionadas em como construir a paz, representando 38,9\%, e as demais dúvidas sobre os componentes da paz, como mostra o Gráfico 1.

Gráfico 1 - Principais dúvidas/comentários dos alunos da escola participante do projeto no programa sobre cultura de paz, Picos, Piauí, 2016
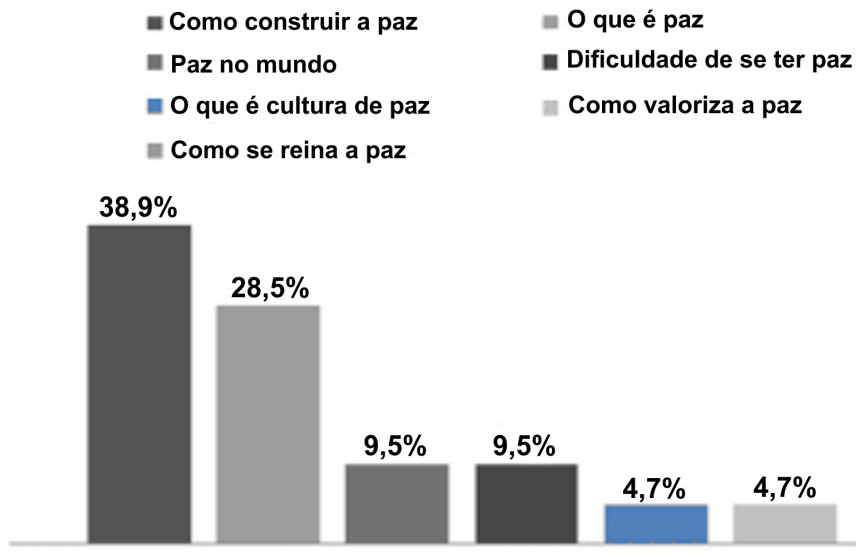

$28,5 \%$
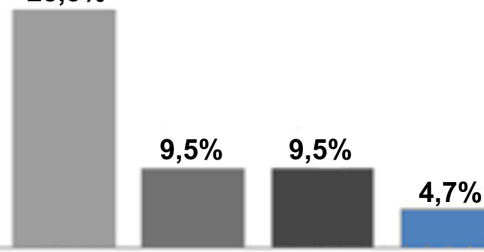

$4,7 \%$

$4,7 \%$

Dúvidas/Comentários

Fonte: Dados da pesquisa (2016).

No dia da transmissão do programa sobre bullying foi averiguado que entre as principais dúvidas/comentários feitas pelos alunos participantes prevaleceram as que abordavam o que fazer para o bullying náo acontecer (33,3\%), seguidas por sua definição e consequências (Gráfico 2). 
Gráfico 2 - Principais dúvidas/ comentários dos alunos da escola participante do projeto no programa sobre bullying, Picos, Piauí, 2016
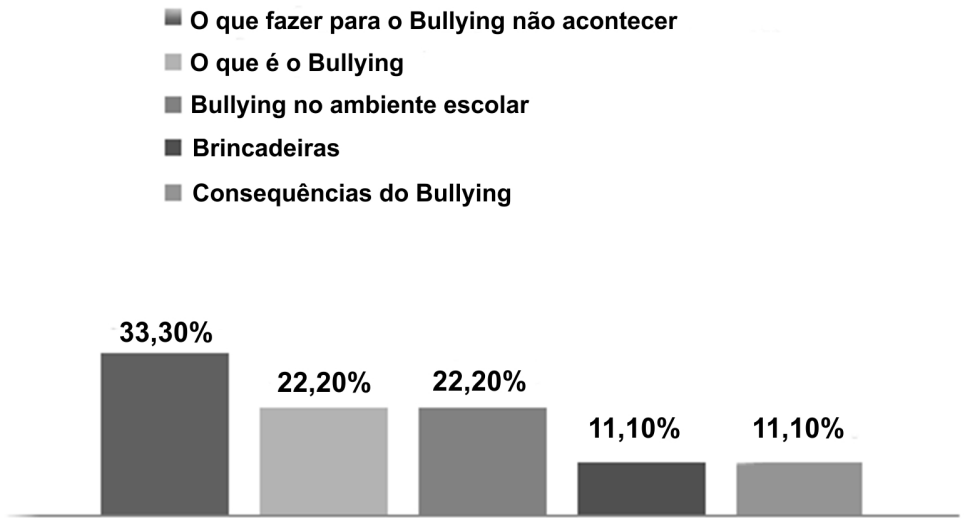

Dúvidas/Comentários

Fonte: Dados da pesquisa (2016).

O debate dessas temáticas com jovens foi relevante, devido às constantes situaçóes em que eles se deparam com esses temas, pois comumente, nos meios de comunicação, vemos casos de violência junto a esse público, principalmente o bullying. Sendo assim, por meio dessas atividades de extensão, a juventude escolar discutiu sobre o tema, inclusive sobre como agir quando tal prática é exercida.

\section{CONSIDERAÇÕES FINAIS}

Projetos de extensôes como o "Web Cuidado em Infância e Juventude nas Escolas" e a "Web Rádio AJIR" representam um aprendizado de enorme valia, pois foi percebido que a web rádio representou uma ferramenta inovadora que despertou um diálogo com interesse de aprendizado pelos jovens participantes com valiosa associação entre tecnologias, saúde e educação.

A web rádio se mostra como um potente meio de troca de saberes, entre usuários e ouvintes. E por meio dessa pesquisa, demonstrouse ainda que se trata de uma forma de levar informação para além dos muros físicos da Universidade, permitindo, inclusive, a parceria entre projetos de extensão entre instituiçóes de diferentes gestores e 
de distintos estados no Brasil.

Nas atividades desenvolvidas, identificou-se um desencaminho de conhecimentos sobre os temas cultura de paz e bullying por parte de alguns alunos quando confrontados com o esperado pelos integrantes do projeto, ressaltando, ainda mais, a importância de explanaçóes de conteúdos sobre as temáticas no ambiente escolar e a implementação de aparelhos virtuais no aprendizado, que pode despertar, sob a perspectiva de uma união com outras práticas e saberes, uma ação humanizada e dinâmica.

\section{REFERÊNCIAS}

ARAÚJO, M. M. S. Violência escolar: sobre métodos e atitudes utilizadas dos professores do ensino fundamental menor em uma escola municipal de Castanhal, 2017. 45 f. Monografia (Pedagogia) - Faculdade de Pedagogia, Universidade Federal do Pará, Castanhal, 2017.

BRASIL. Ministério da Saúde. Comissão Nacional de Ética em Pesquisa. Conselho Nacional de Saúde (BR). Diretrizes e normas regulamentadoras de pesquisas envolvendo seres humanos. Brasília, DF, 2012.

Instituto Brasileiro de Geografia e Estatística - IBGE. Censo Demográfico 2016. Disponível em: <https://cidades.ibge.gov.br/ brasil/pi/picos/panorama>. Acesso em: 14 set. 2018.

CERDAS-AGÜERO, E. Desafíos de la educación para la paz hacia la construcción de una cultura de paz. Revista Electrónica Educare, Heredia, v. 19, n. 2, p. 135-154, maio-ago. 2015. doi: http://dx.doi. org/10.15359/ree.19-2.9.

CORREIA, R. L.; SANTOS, J. G. A importância da tecnologia da informação e comunicação (TIC) na educação a distância (EAD) do ensino superior (IES). Aprendizagem em EAD, Taguatinga, v. 2, n. 1, p. 1 - 16, nov. 2013. Disponível em: <https://portalrevistas.ucb.br/ index.php/raead/article/view/4399/2899>. Acesso em: 14 set. 2018.

DOURADO, I. F. et al. Uso das TIC no ensino de ciências na educação básica: uma experiência didática. Rev. Ed. Ens. Ciênc. 
Human., Londrina, v. 15, n. esp, p. 357-365, 2014. doi: http://dx.doi. org/10.17921/2447-8733.2014v15n0p\%25p

GIL, A. C. Como elaborar projetos de pesquisa. São Paulo; Atlas, 2010. $176 \mathrm{p}$.

Herrera, M. A.; FERnÁNDEZ, D. C.; SEGUEL, R. C. Percepción de los profesores sobre integración de TIC en las prácticas de enseńanza en relación a los marcos normativos para la profesión docente en Chile. Ensaio: aval. pol. públ. Educ., Rio de Janeiro, v. 26, n. 98, p. 163-184, 2018. doi: http://dx.doi.org/10.1590/s010440362017002501119 .

MOTA, D. N. et al. Tecnologias da informação e comunicação: influências no trabalho da estratégia Saúde da Família. J. Health Inform., Sáo Paulo, v. 10, n. 2, p. 45-49, abr.-jun. 2018. Disponível em: < http://www.jhi-sbis.saude.ws/ojs-jhi/index.php/jhi- sbis/article/ viewFile/563/330>. Acesso em: 18 jun. 2018

NAVES, E. T. Fazer-saber: reflexões sobre a função acadêmica da extensão universitária. Em Extensáo, Uberlândia, v. 14, n. 1, p. 9-29, jan.-jul. 2015. doi: http://dx.doi.org/10.14393/REE-v14n12015_ $\operatorname{art} 01$.

OLWEUS, D. School bullying: development and some important challenges. Annu. Rev. Clin Psychol, Palo Alto, v. 9, p. 751-780, 2013. doi: 10.1146/annurev-clinpsy-050212- 185516.

PESSONI, A.; GOULART, E. Tecnologias e o ensino na área da Saúde. ABCS Health Sci., Santo André, v. 40, n. 3, p. 270- 275, 2015. doi: https://doi.org/10.7322/abcshs.v40i3.807.

PINTO, L. W.; ASSIS, S. G. Estudo descritivo das tentativas de suicídio na população idosa brasileira, 2000- 2014. Ciência \& Saúde Coletiva, Rio de Janeiro, v. 20, n. 6, p. 1681-1692, 2015. doi: http:// dx.doi.org/10.1590/1413-81232015206.03532015.

ROCHA, L. A. et al. Dialogando com a juventude sobre o câncer de mama e o câncer de colo do útero. In: SILVA, A. R. V. et al. Interlocuçóes entre vivências coletivas na promoçáo da saúde. Teresina: EDUFPI, 2018. p. 362- 373. 
ROCHA, M. O.; COSTA, C. L.; PASSOS NETO, I. Bullying e o papel da sociedade. Cadernos de Graduaçáo: Ciências Humanas e Sociais, Acaraju, v. 1, n. 16, p. 191-199, 2013. Disponível em: $<$ https://periodicos.set.edu.br/index.php/cadernohumanas/article/ view/534/259>. Acesso em: 14 set. 2018.

RUÍZ, CA. Teología y cultura de paz. Cultura de Paz, Manágua, v. 20, n. 62, p.16-24, 2014. Disponível em: < http://revistasnicaragua. net.ni/index.php/culturadepaz/article/view/1001>. Acesso em: 19 ago. 2018.

SANTOS, A. L. L. et al. Cibercultura e jovens: um diálogo a partir da Psicologia. Revista Mangaio Acadêmico, João Pessoa, v. 2, n. 3, p. 101-106, 2017. Disponível em: <http://revistaadmmade.estacio. br/index.php/mangaio/article/view/4478>. Acesso em: 19 ago. 2018.

TORRES, R. A. M. et al. Comunicação em saúde: uso de uma web rádio com escolares. J. Health Inform., São Paulo, v. 7, n. 2, p. 58- 61, 2015. Disponível em: <http://www.jhi-sbis.saude.ws/ojs-jhi/index. php/jhi-sbis/article/view/325>. Acesso em: 20 jul. 2018.

Submetido em 28 de julho de 2018.

Aprovado em 14 de setembro de 2018. 